\title{
STOCHASTIC DATA IN ASTRONOMY. II. SEARCH FOR HARMONIC COMPONENTS OF TIME SERIES WITH VERY LARGE GAPS
}

\author{
A. F. Kholtygin, ${ }^{1}$, A. B. Shneiwais, ${ }^{1}$ T. E. Burlakova, ${ }^{2}$ and Yu. V. Milanova ${ }^{1}$
}

This is an analysis of certain aspects of using the CLEAN algorithm for Fourier analysis of short segments of time series and of time series consisting of short segments of length $\Delta T$ separated by very long irregular gaps. It is assumed that the time series contain a harmonic component of amplitude A with a period longer than the length of the longest of the segments of the time series plus white noise with dispersion $N^{2}$. Reliability plots are constructed for determining the ranges of the parameters $(V, \varphi)$ for which the CLEAN procedure can be used to determine the values of $v$, the frequency, and $\varphi$, the phase of the harmonic component, with a given accuracy. The results of this analysis are used to search for harmonic components in the variation of the $H \beta$ line profile in spectra of the triple star $\delta$ Ori A obtained in 2004 with the BTA telescope at the Special Astrophysical Observatory of the Russian Academy of Sciences.

Keywords: time series: Fourier analysis: hot stars: line profiles: variability

\section{Introduction}

The most effective way of studying the structure of astronomical objects is to made spectral observations over a long period with high time resolution. In the course of the observations, the integrated flux $F(t, \lambda)$ from the object under study is measured over time intervals $(t, t+\Delta T)$ within a wavelength interval $(\lambda, \lambda+\Delta \lambda)$. Because of random errors in the measurements, the observed flux is a two dimensional random function.

The fluxes $F\left(t_{i}, \lambda_{j}\right)$ are measured for a discrete set of observation times $\left\{t_{i}\right\}$ and a set of wavelengths $\left\{\lambda_{j}\right\}$, where the specific values of $t_{i}$ and $\lambda_{j}$ are determined by the stellar magnitude of the object, the conditions under which it is being observed, and the properties of the detectors (spectrographs and CCD detectors). Because of the finite duration of the time interval $\Delta t$ for each observation (exposure), the choice of the $t_{i}$, which we shall take to be the midpoint of the exposure interval, is of some importance.

(1) Institute of Astronomy, St. Petersburg State University, Russia; e-mail: afk@ theor1.astro.spbu.ru

(2) Special Astrophysical Observatory, Academy of Sciences, Russia

Original article submitted December 31, 2006; accepted for publication February 15, 2007. Translated from Astrofizika, Vol. 50, No. 2, pp. 281-297 (May 2007). 
The set of all values $F\left(t_{i}, \lambda_{j}\right)$ is known as the dynamic spectrum of the object under study. One of the main problems in analyzing dynamic spectra is to identify periodic components in the variation of line profiles. Solving this problem reduces to analyzing the time series $F\left(t_{i}, \lambda_{j}\right)$ for all values of $\lambda_{j}$ lying within the confines of the line profile.

When the length of the time series is sufficient, identifying harmonic components in the variations of line profiles is a routine task [1,2]. Unfortunately, it is not always possible to obtain long time series because of weather conditions and difficulties in getting the required amount of observation time. For these reasons the observed time series are too short or consist of short segments of time series separated by long temporal gaps. Here it often happens that the period of the harmonic variations in the line profiles are longer than any segments of the series.

This paper examines the question of how accurately the characteristics of the harmonic components of the variations in line profiles can be determined, under the assumption that these components are actually present in a series that is being studied. In the second section we state the problem in a more precise form. Section 3 is a preliminary analysis of model time series which contain harmonic components with periods longer than the length of a time series or of the segments of that time series. Model short series and series with long gaps are analyzed by constructing reliability plots [3] in Section 4. In Section 5 the proposed method is used for analyzing some observations of the triple system $\delta$ Ori A made with the BTA telescope in 2004. The conclusions of this paper are summarized in a final section.

\section{Statement of the problem}

We assume that a series of measurements have yielded values $F\left(t_{i}, \lambda_{j}\right)$ of the fluxes from the observed object, with $i=1, \ldots, m$ where $\mathrm{m}$ is the number of observations made and $j=1, \ldots, n$ where $n$ is the number of wavelengths $\lambda_{j}$ within the profile of the line being studied. In order to search for harmonic components of the variation of the line profiles let us construct a set of centered time series $G\left(t, \lambda_{j}\right)=F\left(t, \lambda_{j}\right)-\bar{F}\left(\lambda_{j}\right)$, where $\bar{F}\left(\lambda_{j}\right)=\left[\sum_{i=1}^{m} F\left(t_{i}, \lambda_{j}\right)\right] / m$ is the average flux at wavelength $\lambda_{j}$ over the entire time of the observations. For the CCD matrices customarily used in flux measurements, we can assume that the time series $G\left(t, \lambda_{k}\right)$ and $G\left(t, \lambda_{l}\right)$ are independent for $k \neq l$.

We assume that the function $G\left(t, \lambda_{j}\right)$ can be represented as a combination of harmonic components and white noise:

$$
G\left(t, \lambda_{j}\right)=\sum_{k=1}^{L} A_{k} \cos \left(2 \pi v_{k} t+\varphi_{k}\right)+\sigma_{N} \mathcal{N}
$$

where $A_{k}, v_{k}$, and $\varphi_{k}$ are, respectively, the amplitudes, frequencies, and phases of the harmonic components (which depend on the chosen value of $\left.\lambda_{j}\right), L$ is the number of harmonics, $\mathcal{N}$ is a normally distributed random quantity with zero mathematical expectation and unit dispersion, and $\sigma_{N}$ is the standard deviation of the white noise. Let $A_{\max }=\max \left(A_{k}\right)$, with $k=1, \ldots, L$, be the maximum amplitude of the harmonic components.

We introduce the quantity $\sigma_{N}=A_{\max } / \mathrm{U}$, where $\mathrm{U}=A_{\max } / N$ is a characteristic of the degree to which the noise component contributes to the signal being studied. $\mathrm{U}>>1$ corresponds to the case of a small contribution from the white noise. 
Two problems can be formulated. The first is to identify the harmonic components at a given level of significance $q<<1$ for the hypothesis of a strong peak in the noise component in the real series of observations. The second is the same as the first, but for model time series on a time grid determined by the real series with model harmonic components and with parameters for the harmonic components obtained by solving the first problem. In this case the solution of the model problem should confirm the reliability of the isolation of harmonic components from the real series at a given level of significance. This method has been described in more detail elsewhere [3]. In this paper we restrict ourselves to the case where a single harmonic component is present in model time series $(L=1)$.

For the harmonic analysis we shall use the CLEAN Fourier-analysis algorithm [1] with a modification proposed in Ref. 2.

\section{Fourier analysis of model time series.}

3.1. Short time series. Let us construct a model time series including a harmonic component with a period longer than the length of the time series using the parameters for observations of the triple star $\delta$ Ori A we made with the BTA

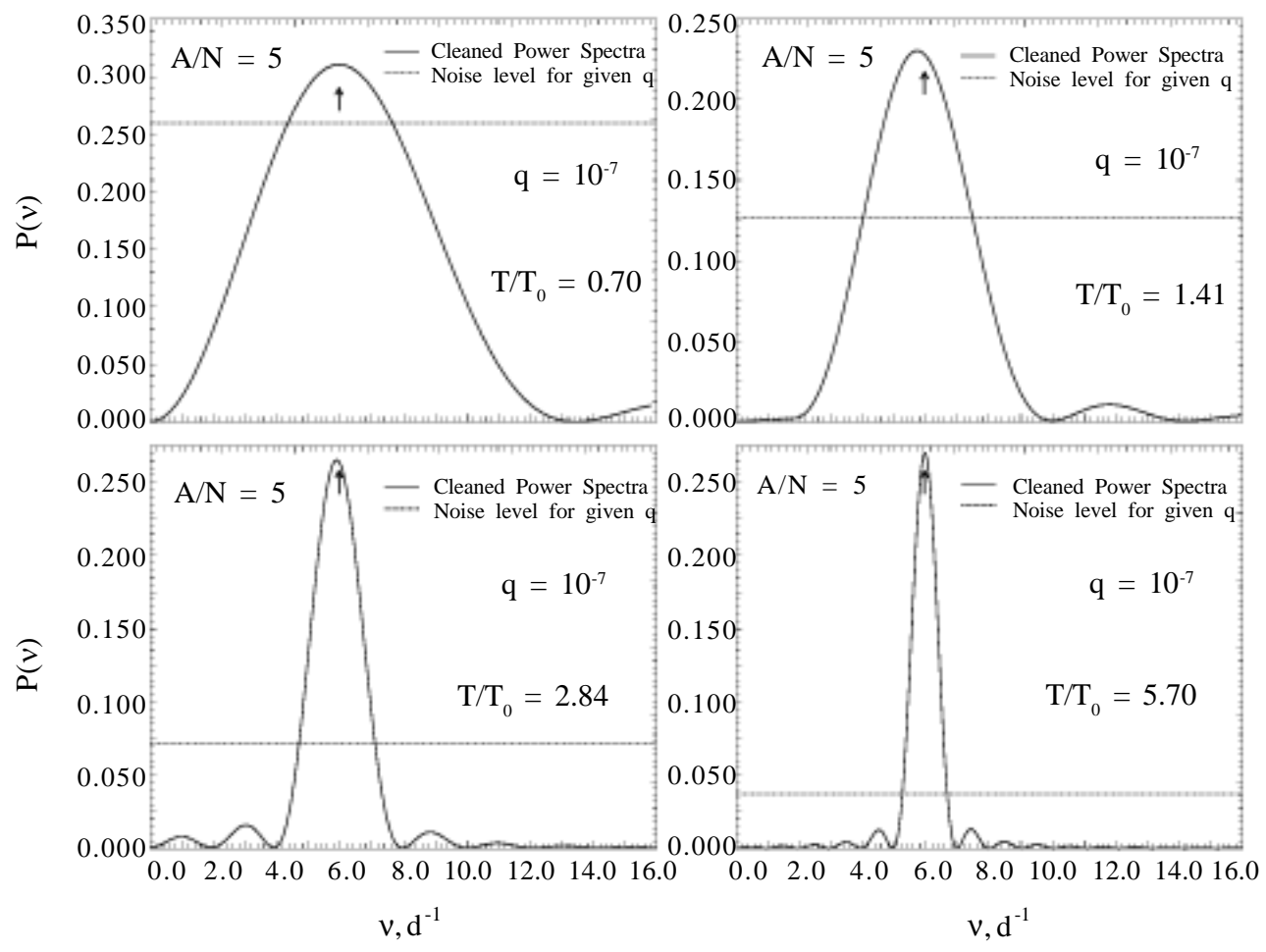

Fig. 1. Periodograms (Fourier power spectra) of model time series with frequency $v_{0}=5.96 \mathrm{~d}^{-1}$ (period $T_{0}=4.03 \div$ ), amplitude $A=1.0$, and phase $\varphi=5.5$ with a step size of $\Delta T=0.003 \mathrm{~d}$ for $N=40,80,160$, and 320 points in the time series (smooth curve) and the noise level corresponding to a significance level $q=10^{-7}$ for the hypothesis that a strong white-noise peak is present in the periodogram (dotted line). The overall durations $T$ of the segments of the time series are indicated in each frame. The arrows denote the exact value of the frequency of the model series. 
telescope in 2004 [4]. We shall assume that over the entire observation time $T, N$ observations are made with a constant time $\Delta T$ between them. We take $T=0.003$ day $=4.3 \mathrm{~min}$, which corresponds roughly to 334 observations over a day. This value of $\Delta T$ is equal to the interval between the successive spectral observations of the triple star $\delta$ Ori A on the BTA in 2004. (See Section 5.)

Figure 1 shows the results of our analysis of model time series with frequency $v_{0}=5.96 \mathrm{~d}^{-1}$ (period $T_{0}=0.168 \mathrm{~d}$ ) for $\mathrm{N}=40,80,160$, and 320 .

The resolution of a periodogram is usually assumed to be equal to $1 / T$ [2]. Thus, from a formal standpoint the minimum frequency which can be found by analyzing a time series segment of length $T$ is $T-v_{\min }=1 / T$. For $N=40$ the length of the time series is $T=0.12 \mathrm{~d}$, which yields $v_{\min }=8.3 \mathrm{~d}^{-1}$. At the same time, it can be seen from Fig. 1 that although the condition $v>v_{\min }$ is not satisfied, the frequency of the periodic component has been determined quite accurately.

Note that for phases of the periodic component which differ from the value $\varphi=5.5$ we have used (e.g., for $\varphi \in[3,5]$ ), the CLEAN method is able to resolve the harmonic component of the time series with $N=40$. We thereby conclude that even for short segments of time series, shorter than the length of the harmonic component of the series, for certain values of the phase of this component its frequency can be determined. This point is discussed in more detail in Section 4.1 of this paper.

An improvement in the frequency resolution can be seen in Fig. 1 as the ratio of the series length $T$ to the period $T_{0}$ increases. However, even for a short time series $(N=40)$, the frequency of the harmonic component is found quite accurately. Figure 2 compares the model and recovered (over a time interval of $T=1.1 \mathrm{~d}$ ) time series. It can be seen that even for time intervals far from the analyzed time series, the time shift of the recovered series is only $0.1-0.2$ periods.

3.2. Analysis of series with very large gaps. Let us analyze the results of a determination of the characteristics of a time series for observations consisting of short (less than the period $P=\mathrm{T}_{0}$ ) segments of series separated by long intervals. We assume that the time series to be analyzed consists of a set of $\mathrm{m}$ short segments of length $T<T_{0}$ separated by large intervals of duration $\Delta T \gg T$.

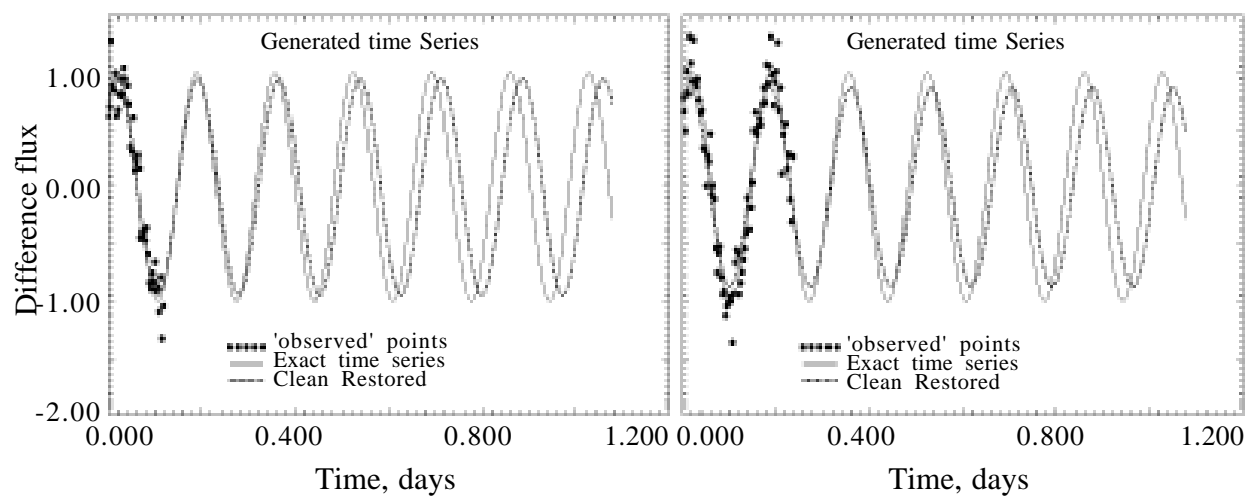

Fig. 2. A comparison of the model series (without a contribution from a noise component $N$ ) and the series recovered from the first 40 (left) and 80 (right) points of the model with $A / N=5$ over a time interval $T=1.1 \mathrm{~d}$. The parameters of the model series are given in the caption to Fig. 1. 


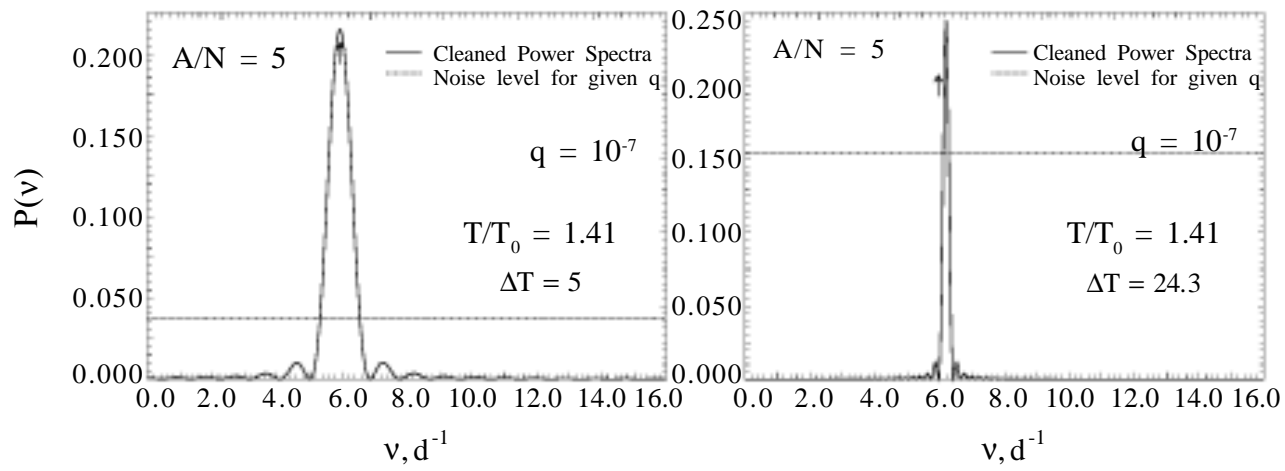

Fig. 3. Same as Fig. 1, but for two sequences $(m=2)$ of time points with $N=40$, separated by intervals of $\Delta T=5 \mathrm{~d}$ and $\Delta T=24.3 \mathrm{~d}$.

Figure 3 shows the results of an analysis of these model time series with $m=2$ and $N=40$ for interval durations $T=5 \mathrm{~d}$ and $24.3 \mathrm{~d}$ using the CLEAN method. As the time interval between the observations increases, the lobe of the periodogram corresponding to the given harmonic component becomes narrower.

\section{Reliability function for time series}

We now describe the method for constructing reliability plots for determination of the harmonic parameters of time series following our earlier paper [3].

Suppose that applying the CLEAN algorithm to the time series being analyzed has yielded components with parameters $v, A^{0}$, and $\varphi$. Let us construct a sequence of model time series with a fixed value $A^{0}$ of the amplitude of the harmonic component and values of $v$ and $\varphi$ within the intervals $v_{\min } \leq v \leq v_{\max }$ and $\varphi_{\min } \leq \varphi \leq \varphi_{\max }$, chosen so as to encompass all possible values of $v$ and $\varphi$ for the given process. In particular, the values of $\varphi$ lie within the range $0 \leq \varphi \leq 2 \pi$.

On the other hand, we restrict the frequencies of the harmonic component to the interval $v \in[0.05,10]$. This interval encompasses all the possible values for the harmonic components of the variations we are studying in the line profiles of stars in early spectral classes associated with rotational modulation of the profiles and nonradial pulsations (NRP) of these stars [5-12].

A model time series is analyzed for all values in the grid $(v, \varphi)$ using the CLEAN algorithm and parameters $v^{*}$, $A^{*}$, and $\varphi^{*}$ are found that may differ from the input parameters $v, A^{0}$, and $\varphi$. We determine the errors in the determination of the parameters of the model series: $\Delta v=\left|v-v^{*}\right|, \Delta A=\left|A^{0}-A^{*}\right|$, and $\Delta \varphi=\left|\varphi-\varphi^{*}\right|$.

We now choose an accuracy criterion for a determination of a given parameter that reduces to establishing the maximum possible deviation between the exact and determined value of each of the parameters for the process being analyzed. This is, it is assumed that if the errors in determining the parameters $v^{*}, A^{*}$, and $\varphi^{*}$ of the found harmonic component do not exceed the chosen maximum deviations, then this component is present in the process being analyzed. 
If, on the other hand, the error in determining even one of the parameters exceeds the maximum permissible, then it is assumed that this harmonic component cannot be recovered on this time grid with a sufficient degree of accuracy.

We define three criteria for the accuracy of determining the parameters of a time series:

Criterion A: $\left|v-v^{*}\right|<0.1,\left|\left(A^{0}-A^{*}\right) / A^{0}\right|<0.1$ and $\left|\varphi-\varphi^{*}\right|<0.1$,

Criterion B: $\left|\nu-v^{*}\right|<0.2,\left|\left(A^{0}-A^{*}\right) / A^{0}\right|<0.2$ and $\left|\varphi-\varphi^{*}\right|<0.2$,

Criterion $C:\left|v-v^{*}\right|<0.5,\left|\left(A^{0}-A^{*}\right) / A^{0}\right|<0.5$ and $\left|\varphi-\varphi^{*}\right|$ arbitrary.

Here $A^{0}$ is the amplitude of the generated model series. Time is measured in days, frequency in $\mathrm{d}^{-1}$, and phase $\varphi$ in radians. Criteria A and B make it possible to judge the frequency, amplitude, and phase of the unknown periodic process with a fairly high degree of reliability, while criterion $\mathrm{C}$ only says that a periodic process is present in a given time series, but that a reliable determination of the characteristics of this process requires further analysis and, possibly, a larger number of points in the series to be analyzed.

Using these criteria for determining the accuracy with which the parameters $v, A^{0}$, and $\varphi$ are determined, we introduce the reliability function $R(v, \varphi, \mathrm{K}, \mathrm{U}, q)$, defined as

$$
R(v, \varphi, \mathrm{K}, \mathrm{U}, q)= \begin{cases}1, & \text { kriterion } \mathrm{K} \text { satisfied } \\ 0, & \text { kriterion } \mathrm{K} \text { not satifaed }\end{cases}
$$

The values of the reliability function $R(v, \varphi, \mathrm{K}, \mathrm{U}, q)$ depend on the choice of accuracy criterion $\mathrm{K}$, the ratio $\mathbf{U}=A / N$, and the assumed significance level $q$.

We now illustrate the use of the reliability function with the following example. Assume that the Fourier analysis of some time series $F(t)$ has resolved a harmonic component with frequency $v$, amplitude $A^{0}$, and phase $\varphi$. If the value of the reliability function $R(v, \varphi, \mathrm{K}, \mathrm{U}, q)=1$, then we can regard this component as having been resolved in accordance with the accuracy criterion $\mathrm{K}$ at a level of significance $q$. If, on the other hand, $R(v, \varphi, \mathrm{K}, \mathrm{U}, q)=0$, then we shall assume that this component cannot be resolved with the given accuracy. Note, however, that this component might be detected if a less rigorous accuracy criterion $\mathrm{K}$, a larger ratio $A / N$, and (or) a higher level of significance $q$ were specified.

The degree of reliability with which an unknown harmonic component is resolved from a time series at a given level of accuracy is conveniently illustrated with the aid of reliability plots, which indicate the value of the function $R(v, \varphi, \mathrm{K}), q)$ as a function of $v$ and $\varphi$. According to a comment in Ref. 2, it is also desirable to verify whether the frequency of the found harmonic component lies at the maximum of a spectral window near which the reliability of the determination of the parameters of this component decreases.

4.1. Reliability plots for short time series. Reliability plots for the time grids described in Section 3.1 and accuracy criteria A, B, and $\mathrm{C}$ are shown in Fig. 4 for the model series with an added noise component $A / N=5$ and $q=10-7$. The black regions of the figures indicate the ranges of $v$ and $\varphi$ for which the reliability function $R(v, \varphi, \mathrm{K}, q)=1$. Within those regions the parameters of the model series can be recovered with the specified accuracy.

The chosen value of $A / N=5$ is typical for harmonic components of the variation of line profiles in the spectra of hot stars $[4,5,13]$. It should, however, be noted that our calculations have shown that the values of the reliability function only depend weakly on the ratio $A / N$ as far as $A / N \approx 1$, and only for smaller values of this ratio will the region 

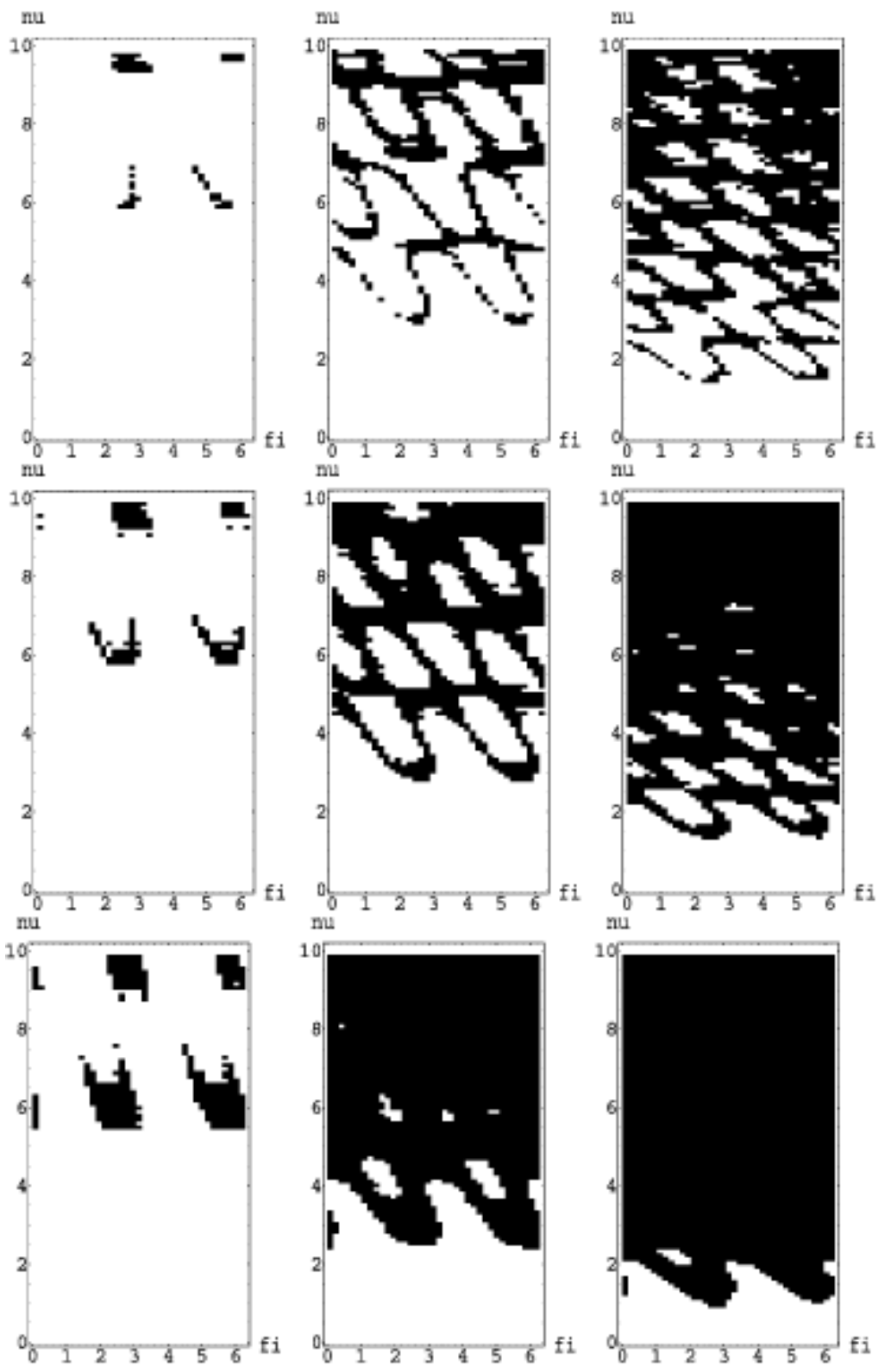

Fig. 4. Reliability plots for determining the parameters $v$ and $\varphi$ by analyzing short time series. Charts A (upper row), B (middle row), and $\mathrm{C}$ (bottom row). In each row the left chart corresponds to a time series of $N=40$ uniformly distributed time readouts with $\Delta T=0.003 \mathrm{~d} \quad\left(v_{\min }=8.3 \mathrm{~d}^{-1}\right)$, the center chart, to $N=80$ $\left(v_{\text {min }}=4.2 \mathrm{~d}^{-1}\right)$, and the right chart, to $N=160\left(v_{\min }=2.1 \mathrm{~d}^{-1}\right)$. The charts have been constructed for a level of significance $q=10^{-7}$ and $A / N=5$. 
of $(\mathrm{v}, \varphi)$ for which $R(\mathrm{v}, \varphi, \mathrm{K}, q)=1$ decrease sharply. When $A / N<5$ this region essentially disappears, which means that it is impossible to resolve regular components from the segments of very noisy time series. Given the weak dependence of the reliability function on the ratio $A / N$ for $A / N \geq 3-5$, in the following we shall only consider reliability plots for $A / N=5$.

Formally, the lower boundary of the range of frequencies of the harmonic components which can be resolved from a time series of duration $T$ is $v_{\min }=1 / T, \mathrm{~d}^{-1}$, where $\mathrm{T}$ is measured in days. (See Section 3.1.) Substituting our values of $N=40,80$, and 160 in this expression, we find, respectively: $T=0.12 \mathrm{~d}, v_{\min }=8.3 \mathrm{~d}^{-1}, T=0.24 \mathrm{~d}, v_{\min }=4.2 \mathrm{~d}^{-1}$, and $T=0.48 \mathrm{~d}, v_{\min }=2.1 \mathrm{~d}^{-1}$.

At the same time, Fig. 4 shows that even when criterion $A$ is used along with a very low value of $q=10^{-7}$, it is possible to recover the parameters of a harmonic component specified on grids with $N=40$, 80 , and 160 with sufficient accuracy in the region $v<v_{\text {min }}$, although only for selected values of $v$ and $\varphi$. For $N=40$, the actual boundary of the region in which the frequencies of harmonic components can be determined is $v_{\min }^{\text {real }} \approx 6 \mathrm{~d}^{-1}$.

Note that, according to Section 3.1, the parameters of the harmonic component of the model time series $(N=40$, $\mathrm{v}=5.96 \mathrm{~d}^{-1}$ ) being analyzed there lie within the region where the reliability function $R(v, \varphi, \mathrm{K}, q)=1$.

Increasing $N$ to $N=80$ and $N=160$ reduces the lower boundary of the frequencies of the harmonic components which can be determined to $\approx 2.5 \mathrm{~d}^{-1}$ and $\approx 1.5 \mathrm{~d}^{-1}$, respectively, but does not eliminate the patchiness in the reliability chart. This indicates that there are large ranges of the parameters $(\nu, \varphi)$ within which the parameters of harmonic components cannot be resolved in any way.

The presence of such forbidden regions of $(v, \varphi)$ is related both to the forced centering of the time series and to the extremely low value of $q=10^{-7}$ that we have used in constructing the reliability plot. A less rigorous criterion for resolving a harmonic component (going from criterion A to criteria B and C) leads to the almost complete disappearance of the lacunae in the reliability plots at frequencies $v>v_{\min }$ for $N \geq 80$. At the same time, for a short segment of a time series with $v>v_{\text {min }}$, even when criteria $\mathrm{B}$ and $\mathrm{C}$ are used the region within which the parameters $(N \geq 80)$ are reliably determined remains extremely small.

4.2. Reliability plots for short segments of time series separated by long gaps. Figure 5 shows the reliability plots for the time grids described in Section 3.2 with a large gap $\Delta T$, the accuracy criteria A, B, and C for model series, a noise component $A / N=5$, and $q=10^{-7}$. Our calculations show that adding white noise causes essentially no change in the region of reliably determined parameters for the harmonic component compared to the case without a noise component, $A / N=\infty$.

It is clear from this figure that, although the range of $\nu, \varphi$ where the parameters of the harmonic component can be determined with a given accuracy is considerably greater than the same region for a time series consisting of a single segment, the regions of undetermined parameter values remained very large. Note that adding an additional segment of the series, although separated by a large gap, leads to reduction in the minimum frequency for which it is still possible to resolve a model component in the model time series. 

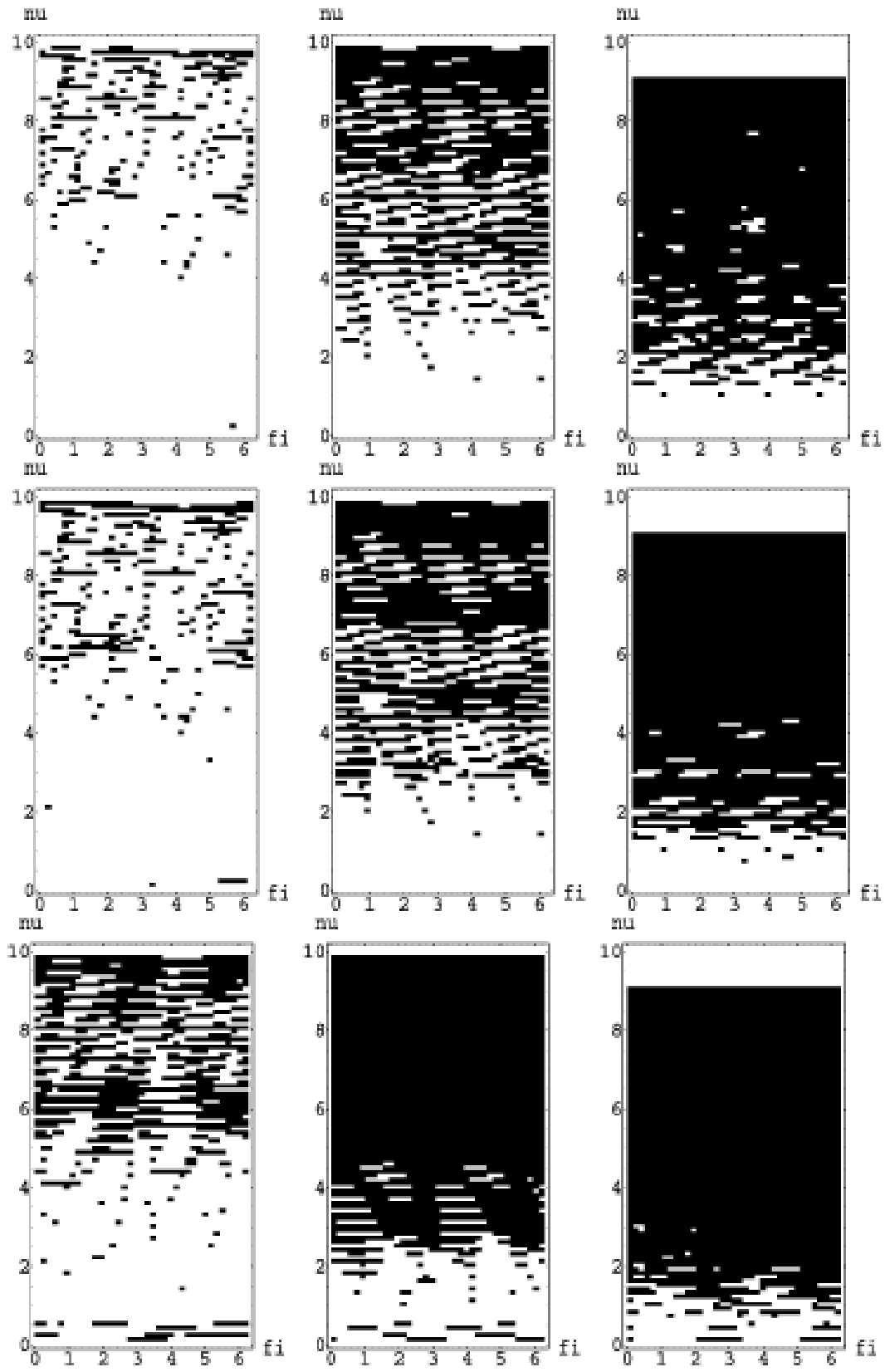

Fig. 5. Reliability plots for determining the parameters $v$ and $\varphi$ for series with a large gap $T_{g a p}=3 \mathrm{~d}$. Charts A (upper row), B (middle row), and C (bottom row). In each row the left chart corresponds to a time series of $N=40+40$ uniformly distributed time readouts with $\Delta T=0.003 \mathrm{~d}$, separated by a gap $T_{g a p}$, the center chart, to $N=80+80$, and the right chart, to $N=160+160 . q=10^{-7}$ and $A / N=5$. 


\section{Search for regular components of the variations in line profiles in the spectrum of the star $\delta$ Ori $A$}

We made spectral observations of the triple system $\delta$ Ori A as part of a program to search for rapidly varying line profiles in the spectra of stars in the early spectral classes [5] on the night of January 10-11, 2004 on the 6-m telescope at the Special Astrophysical Observatory. The NES quartz echelle spectrograph, fixed-mounted in the Naismith focus with a $2048 \times 2048$ pixel CCD detector (Uppsala CCD), was used. The characteristics of the measurements and the parameters of the star are described elsewhere [4].

Over the entire observation time of $\approx 2^{\mathrm{h}} 50^{\mathrm{m}} \quad 40$ spectra of the stars were obtained with an exposure of $180 \mathrm{~s}$. Taking the readout time of the CCD into account, the time resolution was $260 \mathrm{~s}$. The time interval between successive spectra of the star was the $\Delta T=0.003 \mathrm{~d}$ we have used in constructing the model time series.

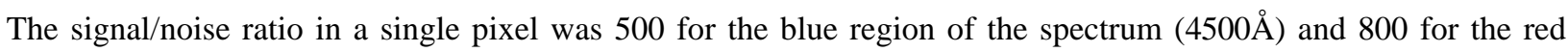
(6000A). The CCD images of the echelle spectra were initially reduced using MIDAS. To study the variability in the line profiles, the processed spectra were normalized to the continuum constructed in each echelle order. Difference profiles (individual line profile minus the average profile) were constructed for resolving variable details of the line profiles. The average profile was obtained using all 40 spectra of $\delta$ Ori A.

A preliminary analysis [4] of the spectra of $\delta$ Ori A revealed the possible presence of a harmonic component with a frequency of $v \approx 5.9 \mathrm{~d}^{-1}$ in the variations of the HeII $\lambda 4686$, HeI $\lambda 4713$, and $\mathrm{H} \beta$ lines. In this section we examine the
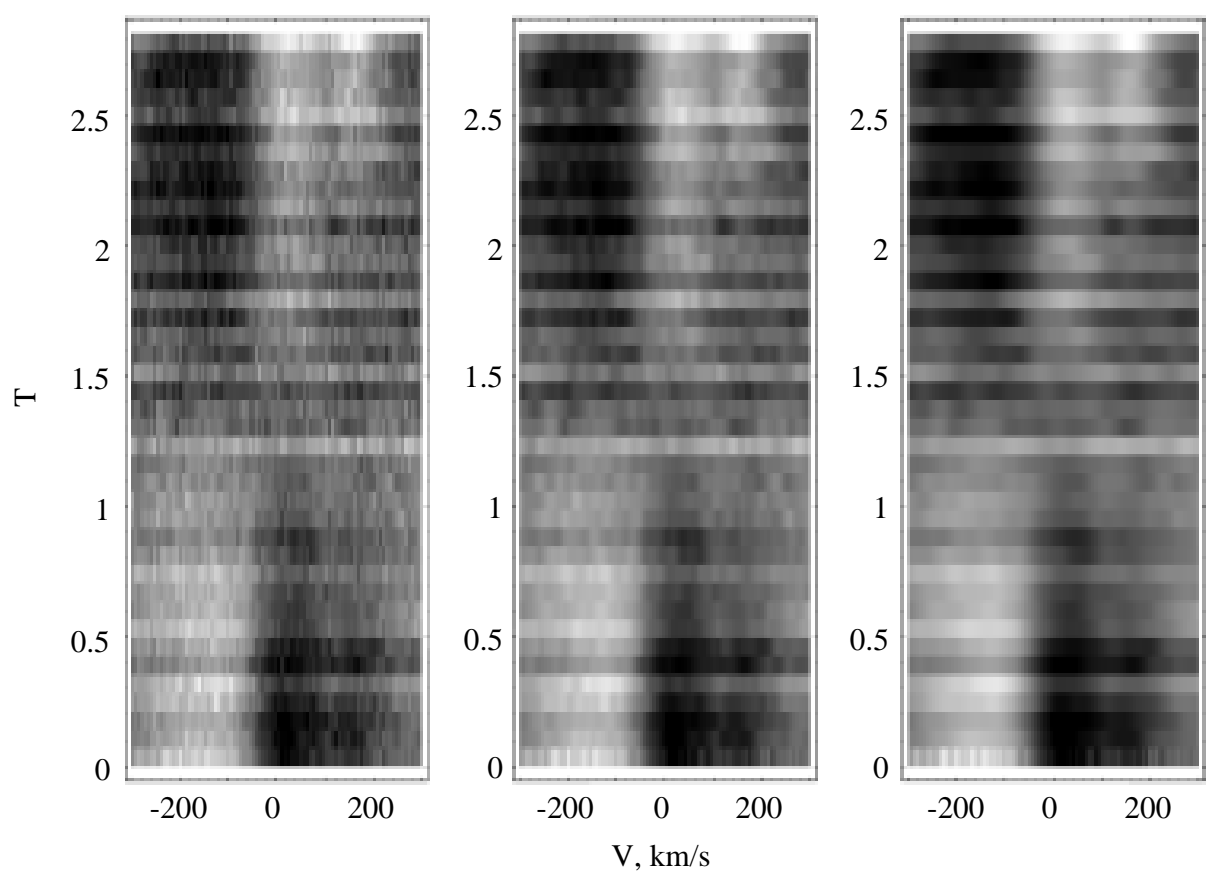

Fig. 6. Dynamic spectra of the variations in the $\mathrm{H} \beta$ line profile in the spectrum of $\delta$ Ori A. (Left) unsmoothed variations in the profiles, (center) smoothed with a gaussian filter of width $0.1 \AA$, and (right) with a width of $0.2 \AA$. The interval between successive spectra was $4 \mathrm{~min}$. The time axis (hours) is directed upward and the radial velocities are in $\mathrm{km} / \mathrm{s}$. The dark regions in the figures correspond to less bright portions compared to the average profile (valleys) and light regions, to brighter portions (humps). 
reliability with which the harmonic component was resolved using the reliability function method described in Section 4. For greater reliability of the analysis, we concentrate on analyzing the variations in the H $\beta$ line profiles, since these variations were greatest for that line.

As an illustration, Fig. 6 shows some dynamic difference profiles of the $\mathrm{H} \beta$ line in the spectrum of $\delta$ Ori A. The deviations of the individual profiles from the average is shown in all the spectra by grey shading. For clarity the wavelength scale has been converted into Doppler shifts from the line center. The left frame shows the original variations in the profiles, while the center and right frames show the data as smoothed by gaussian filters of width $W=0.1$ and $0.2 \AA$, respectively.

Regular variations in the profiles of the $\mathrm{H}$ line can be seen in these figures. The humps of the profiles (the light regions in the density plot) shift from violet to the red wing of the profile. When the filter width is increased, the regularity of the variations in the profile show up more distinctly.

In order to search for regular components of the variations in the profiles we have carried out a Fourier analysis of the variability in line profiles in the spectrum of $\delta$ Ori A. A modification [2] of the CLEAN algorithm [1] was used
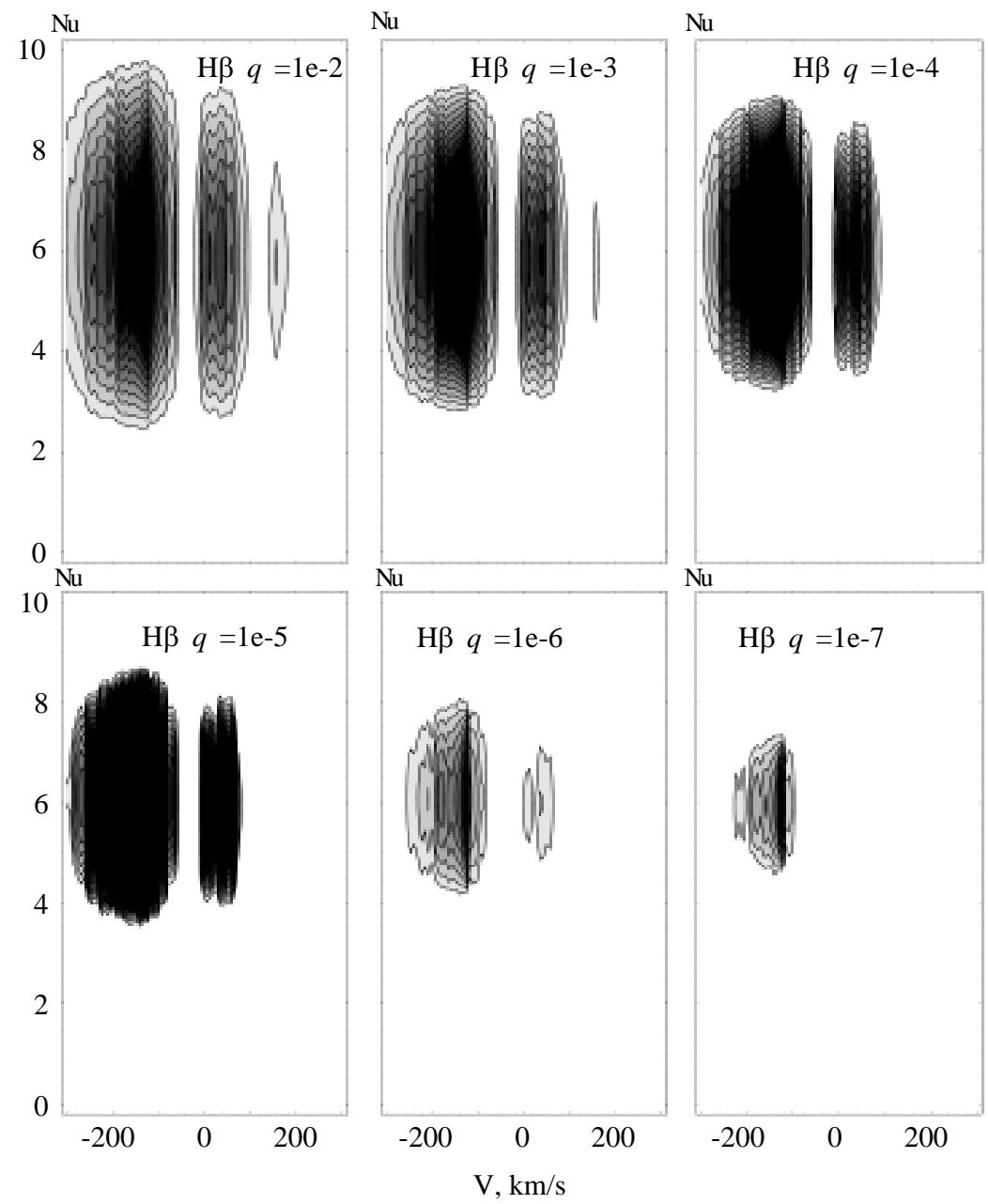

$\mathrm{Nu}$

$\mathrm{H} \beta q=1 \mathrm{e}-7$

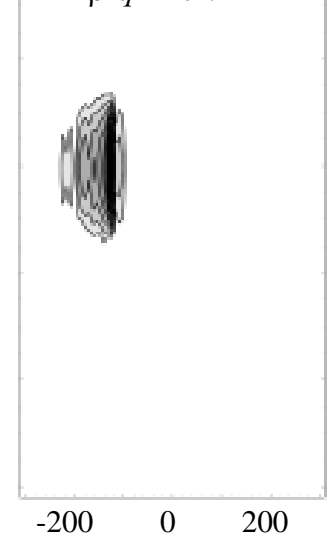

Fig. 7. Fourier power spectra of the variations in the $\mathrm{H} \beta$ line profiles in the spectrum of $\delta$ Ori A for significance levels $q=10^{-2}-10^{-7}$. 
to remove false peaks from the Fourier spectrum.

Figure 7 shows contour plots of the square of the modulus of the amplitude of the Fourier transform (Fourier spectra) for the $\mathrm{H} \beta$ line with different significance levels $q$ for the hypothesis that a strong peak is present in the periodogram of the noise component of the time series. To each level of significance there is a corresponding value of the number $N_{q}$ of readouts in the periodogram such that the probability that the maximum value of the white noise in the periodogram exceeds the level $N_{q}$, as well as the value of $q$. In constructing the density diagram for the Fourier power spectrum, all values in the periodogram less than $N_{q}$ corresponding to the significance level $q$, were eliminated. Only readouts of the periodogram significant at a level $q$ are thereby included in the plots.

In addition, in constructing the periodograms we have only used those values of the frequencies $v$ and Doppler shifts $V$ for which the reliability function $R(\mathrm{v}, \varphi, \mathrm{K}, q)=1$, where $\varphi$ is the phase of the harmonic component corresponding to a given value of $V$.

A broad peak at frequency $v \approx 5.9 \mathrm{~d}^{-1}$ can be seen in the figure. The large width of this peak is related to the comparatively narrow resolution of the Fourier spectrum owing to the small duration of the observation period, as can be seen clearly in Fig. 1. As $\mathrm{q}$ is reduced, $N_{q}$ increases, while there is a reduction in the width of the peak in the periodogram corresponding to the resolved harmonic component.

$P=1 / \mathrm{v} \approx 4^{\mathrm{h}} .1$ is greater than the duration $T \approx 2^{\mathrm{h}} 50^{\mathrm{m}}$ of the observations, so it cannot immediately be identified with a period of regular variations in the profiles. Thus, we shall refer to this quantity as the quasi-period of the regular variations in the profiles.

It is usually assumed that the accuracy with which a frequency of the harmonic component is determined is $\Delta v=1 / T$, which is greater than $v$ itself. At the same time, our analysis given above shows that when a harmonic component with frequency $v \approx 5.9 \mathrm{~d}^{-1}$ is present in the time series being analyzed, for those frequencies and phases such that the reliability function $R(v, \varphi, \mathrm{K}, q)=1$, its frequency is determined with a greater accuracy corresponding to the chosen criterion $\mathrm{K}$.

As an additional argument in favor of the hypothesis that a harmonic component is present in the variations of the line profiles, we note that the recovered time series well reproduces the behavior of the variations in the H $\beta$ line profile in the real time series, as can be seen clearly in Fig. 8.

At present we can state that the variations in the difference profiles of the $\mathrm{H} \beta$ line in the spectrum of $\delta$ Ori A can be approximated by segments of a sinusoid with frequency $v \approx 5.9 \mathrm{~d}^{-1}$ and, therefore, a period of $P \approx 4$ h .1 , at least for $V=-200 \div 100 \mathrm{~km} / \mathrm{s}$. Figure 7 shows that raising the level of significance $q$ from $10-7$ to $10-2$ leads to an increase in the range of wavelengths (Doppler shifts) in the $\mathrm{H} \beta$ line profile for which the possible presence of a harmonic component with frequency $v \approx 5.9 \mathrm{~d}^{-1}$ can be revealed.

Proceeding from our study of the possibility of determining the parameters of a harmonic component of the variation in line profiles for $N=40$ (Fig. 4), it is obvious that these parameters can be found only within narrow ranges of phase for the harmonic component (and the related ranges of Doppler shifts).

The absence in the Fourier spectrum of maxima associated with a harmonic component with a period of $\approx 4^{\mathrm{h}}$ for Doppler shifts $V$ different from those in Fig. 7 may not be a real effect, but be related to unfavorable values of the phases of this component for Fourier analysis. Thus, we may reach a preliminary conclusion that this harmonic component may 


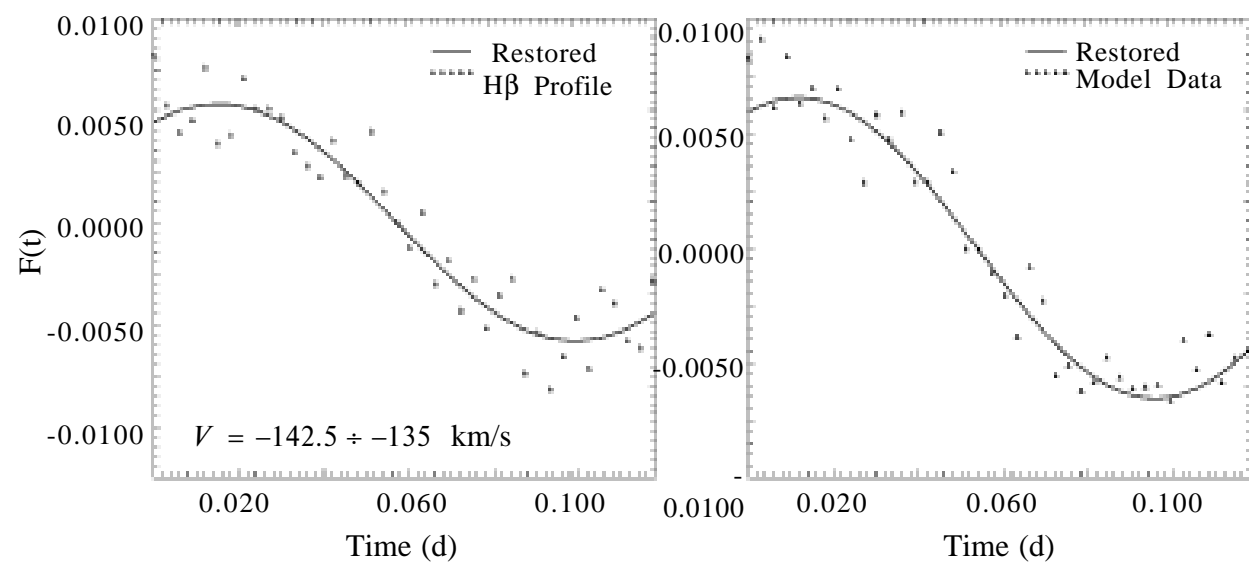

Fig. 8. A comparison of the original time series of the variations in the Ha line profile in the spectrum of $\delta$ Ori A (January 10/11, 2004) with the reconstructed series obtained using the found parameters of the harmonic component and $\mathrm{U}=4$ in the range of Doppler shifts $\mathrm{V}=-142.5$ to $-135 \mathrm{~km} / \mathrm{s}$ from the line center.

appear in the variations of the $\mathrm{H} \beta$ line profiles in the spectrum of $\delta$ Ori A for all Doppler shifts relative to the line center.

Variations in the profiles with a phase of the harmonic component that changes continuously along the profile are typical for the variability of profiles associated with nonradial photospheric pulsations of the star [14]. We have previously [4] found that the variability of line profiles in the spectrum of $\delta$ Ori $A$ is associated with a quadrupole $(l=2)$ mode based on a wavelet spectrum analysis of variations in line profiles in the spectrum of this star. The present studies provide additional evidence for this proposition.

In order to confirm the reality of the period found here, as well as the nature of the observed variations in the line profiles suggested by the authors, it is proposed that spectra of $\delta$ Ori A be taken over 2-3 nights, which would encompass 4-6 cycles of the nonradial pulsations.

\section{Conclusion}

The following conclusions follow from this study of the features of Fourier analysis with the CLEAN algorithm of short time series, shorter than the period of a harmonic component in the series and time series consisting of short segment of time series separated by large gaps, as well as from an analysis of spectral observations of the triple star $\delta$ Ori A:

1. For certain values of the phases of the harmonic component of the time series being analyzed, it is possible to determine its frequency, amplitude, and phase quite accurately based on a Fourier analysis of short segments of time series with lengths shorter than the period of the harmonic component.

2. Fourier analysis of time series consisting of short segments (of length shorter than the period of the harmonic component of the series) separated by long gaps revealed a substantial increase in the range of parameters over which 
the parameters of the harmonic component can be determined compared to analysis with just one short segment of the series.

3. The $\mathrm{H} \beta$ line profile in the spectrum of $\delta$ Ori $\mathrm{A}$ is variable with a variability amplitude of $0.5-1 \%$. Evidence has been obtained of a possible harmonic component with a period of $\approx 4^{\mathrm{h}}$ in the variations of the $\mathrm{H} \beta$ line profile. This component of the variations in the $\mathrm{H} \beta$ line profile may be related to quadrupole-mode $(l=2)$ nonradial photospheric pulsations of stars in the $\delta$ Ori A system.

This work was supported by RFFI grant Nos. 05-02-16995 and 06-02-17096 and a grant from the President of the Russian Federation for support of leading scientific schools, No. NSh-8542-2006.2.

\section{REFERENCES}

1. D. H. Roberts, J. Lehar, and J. W. Dreher, Astron. J. 93, 968 (1987).

2. V. V. Vityazev, Analysis of Nonuniform Time Series [in Russian], Izd. SPbGU, St. Petersburg, p. 68 (2001).

3. A. F. Kholtygin and A. B. Shneiwais, Astrofizika 48, 87 (2005).

4. A. F. Kholtygin, T. E. Burlakova, S. N. Fabrika, G. G. Valyavin, and M. V. Yushkin, Astron. zh., in press (2006).

5. A. F. Kholtygin, D. N. Monin, A. E. Surkov, and S. N. Fabrika, Pis'ma v Astron. zh. 29, 208 (2003).

6. S. Marchenko and A. F. J. Moffat, Astrophys. J. 499, L195 (1998).

7. C. Neiner, A. M. Hubert, M. Floquet et al., Astron. Astrophys. 388, 899 (2002).

8. H. Henrichs, L. Kaper, and J. S. Nichols, Astron. Astrophys. 285, 565 (1994).

9. J. A. de Jong, H. F. Henrichs, S. Schrijvers et al., Astron. Astrophys. 345, 172 (1999).

10. J. A. de Jong, H. F. Henrichs, L. Kaper, J. S. Nichols et al., Astron. Astrophys. 368, 601 (2001).

11. L. Kaper, H. F. Henrichs, A. W. Fullerton et al., Astron. Astrophys. 327, 281 (1997).

12. L. Kaper, H. F. Henrichs, J. S. Nichols et al., Astron. Astrophys. 344, 231 (1999).

13. A. F. Kholtygin, G. Galazutdinov, T. E. Burlakova et al., Astron. zh. 83, 620 (2006).

14. J. H. Telting and C. Schrijvers, Astron. Astrophys. Suppl. Ser. 121, 343 (1997). 